\title{
Heparin isomeric oligosaccharide separation using volatile salt strong anion exchange chromatography
}

\author{
Rebecca L. Miller ${ }^{1,2,3}$, Scott E. Guimond ${ }^{1}$, Maitreyi Shivkumar ${ }^{1,5}$, Jemma Blocksidge ${ }^{1,4}$, James \\ Austin $^{1,4}$, Julie A. Leary ${ }^{2}$, Jeremy E. Turnbull ${ }^{1}$. \\ ${ }^{1}$ Centre for Glycobiology, Department of Biochemistry, Institute of Integrative Biology, University of \\ Liverpool, Crown Street, Liverpool, L69 7ZB, England, UK; \\ ${ }^{2}$ Departments of Molecular and Cellular Biology and Chemistry, University of California, 1 \\ Shields Dr. Davis, CA 95616 USA. \\ ${ }^{3}$ Current address: Department of Oncology, University of Oxford, Old Road Campus, Oxford, \\ OX3 7DQ \\ ${ }^{4}$ Current address: Department of Molecular \& Clinical Cancer Medicine, Institute of \\ Translational Medicine, University of Liverpool, $1^{\text {st }}$ floor Sherrington Building, Ashton Street, \\ Liverpool, England, L69 3GE \\ ${ }^{5}$ Current address: Division of Infection and Immunity, University College London, 1st Floor, \\ Cruciform Building, Gower Street, London, WC1E 6BT.
}

Authors email address: rebecca.miller@oncology.ox.ac.uk

Corresponding Authors: Rebecca L. Miller (rebecca.miller@oncology.ox.ac.uk) and Jeremy E. Turnbull (j.turnbull@liverpool.ac.uk).

\section{Abstract}

The complexity of heparin and heparan sulfate saccharides makes their purification, including many isomeric structures, very challenging and is a bottleneck for structure-activity studies. High resolution separations have been achieved by strong anion exchange (SAX) chromatography on Propac PA1 and cetyltrimethylammonium (CTA)- $\mathrm{C}_{18}$ silica columns but these entail subsequent desalting methodologies, consequent sample losses and are incompatible with orthogonal chromatography methodologies and in particular mass spectrometry. Here we present the CTA- 
SAX purification of heparin oligosaccharides using volatile salt (VS) buffer. In VSCTA-SAX the use of ammonium bicarbonate buffer for elution improves resolution through both weaker dissociation and conformational co-ordination of the ammonium across the sulfate groups. Using ion-mobility mass spectrometry (IMMS) we demonstrate that isomeric structures have different structural conformations, which makes chromatographic separation achievable. Resolution of such structures is improved compared to standard SAX methods, and in addition VSCTA-SAX provides an orthogonal method to isolate saccharides with higher purity. Since ammonium bicarbonate is used, the samples can be evaporated rather than desalted, preventing substantial sample loss and allowing more effective subsequent analysis by electrospray-mass spectrometry (ESI-MS). We conclude that VSCTA-SAX is a powerful new tool which helps address the difficult challenge of heparin/HS saccharide separation, and will enhance structure-activity studies.

\section{Introduction}

Heparin and HS are part of the glycosaminoglycan (GAG) family of sugars. Heparin was first discovered in 1916 and then later developed into an anticoagulant based drug which was first used in 1935. Heparin and HS play an important role in the regulation of biological systems, with chemical synthesis, enzymatic synthesis and oligosaccharide purification are being used to obtain pure structures to understand structure-function relationships ${ }^{1-5}$. Heparin and HS are highly anionic structures, with their synthesis being non-template driven with enzymatic specificity creating variable sulfated regions and acetylated regions identified in HS. Sulfate groups can reside on the 2-O position of the uronic acid group, and the 6-O, 3-O and N-position 
of the glucosamine, with the N-positions also able to adopt an acetate group and occasionally an amine. It is this micro-heterogeneity in sulfation that changes between organs ${ }^{6}$, stage of development $^{7-8}$, communication and environment ${ }^{9}$, providing heparin and HS with a dynamic conformational versatility and thus a vast range of biological functions ${ }^{10}$.

Size exclusion chromatography (SEC) is often the first method used in heparin / HS oligosaccharide purification ${ }^{11}$. SEC separation depends not just on the number of saccharide units, but also on the number of sulfate groups, thus increasing the likelihood of oligosaccharides with different sequences and structures having equivalent hydrodynamic radii ${ }^{12}$. Nevertheless, in isolation of structures ranging from dp4 to dp12, SEC will separate roughly in dp2 increments, due to the disaccharide cleavage specificity of various depolymerisation methods ${ }^{13}$. Further purification of structures isolated by SEC is most commonly achieved using strong anion exchange (SAX).

SAX is a powerful heparin/HS separation method, as it offers higher oligosaccharide resolution over other methods currently available, including hydrophilic interaction chromatography (HILIC) ${ }^{14}$, reverse phase ion pairing (RP-IP) ${ }^{15-16}$, and capillary electrophoresis $(\mathrm{CE})^{17-18}$. With small saccharides (for example disaccharides), there is a significant degree of separation dependant on the presence of 2OS, 6OS and NS groups, but as the oligosaccharide becomes longer this resolution decreases ${ }^{19}$, necessitating the use of additional SAX or orthogonal chromatography methods.

Cetyltrimethylammonium SAX (CTA-SAX) is an alternative SAX method which uses a C8 or C18 column matrix derivatised with $\mathrm{CTA}^{20-21}$. The coating of CTA is dynamic and fine tuning of this coat results in the resolution of oligosaccharides containing up to 20 sulfate groups. 
The extent of CTA derivatisation is determined by the water-methanol ( $\mathrm{v} / \mathrm{v})$ concentration that the column is derivatised in. However, a drawback of this method arises from the use of the ammonium methane sulphonic acid mobile phase at $\mathrm{pH} 2.5$ to create the eluting gradient. This method has not been widely used due to the difficulty in producing the derivatised column, weaker functional end groups and acidic elution.

RP-IP chromatography is also used to separate oligosaccharides, using di-, tri-, butylamines $^{22}$. All eight disaccharides can be separated using a tri-butyl quaternary amine ${ }^{23}$ and for larger oligosaccharides a tri-ethyl amine is generally used as the ion pairing agent ${ }^{24}$. This method has been employed in the analysis of sugars from model organisms, such as Caenorhabditis elegans and Drosophila ${ }^{25}$. It has also been used in the compositional analysis of HS from human tissues, such as liver ${ }^{26}$ and in pharmacokinetics in the analysis of contaminated oral heparin ${ }^{27}$. RP-IP has high resolving capabilities, but removal of the ion pairing reagent is problematic, resulting in oligosaccharide losses through desalting and causing in-source contamination when coupled with a mass spectrometer.

Heparin and HS have polar carboxylates, sulfates and hydroxyl groups and are good candidates for the HILIC technique ${ }^{28}$, where HILIC has been used to separate a wide range of GAG saccharides, as well as O-glycans and N-glycans ${ }^{29-31}$. Recently HILIC LC-MS has been used in the analysis of GAGs from tissues, with studies focusing on CS (chondroitin sulfate) / DS (dermatan sulfate) in connective joint tissues ${ }^{14,32}$ and profiling heparin and $\mathrm{HS}^{33-37}$, using the parent ion and collision induced dissociation (CID) differences. HILIC uses volatile buffers and is compatible with mass spectrometry, but has low oligosaccharide resolution.

In this paper, we describe an ammonium based (volatile salt) CTA-SAX method in conjunction with SEC and conventional SAX that provides improved separation of isomeric 
heparin oligosaccharides. The purification of heparin oligosaccharides using the VSCTA-SAX method avoids the need for desalting, improves column resolution, and the products are directly compatible with mass spectrometry analysis.

\section{Methods}

Materials and Reagents. All chemicals of analytical or HPLC grade purity were purchased from Sigma (Gillingham, UK) and VWR (Lutterworth, UK) unless otherwise stated. Disaccharides standards 1-8 were purchased from Iduron (Manchester, UK). Heparin was purchased from Alfa Asear (Massachusetts, USA). Heparinase I, II, and III enzymes were purchased from IBEX (Canada).

Disaccharide CTA-SAX chromatography. CTA-SAX was performed based on the method of Mourier \& Viskov (2004) utilising a Discovery $\mathrm{C}_{18}$ silica column $(250$ x $4.6 \mathrm{~mm}, 5$ $\mu \mathrm{m}$, Supelco) which was derivatised with $1 \mathrm{mM}$ cetyltrimethylammonium in a water:methanol ratio of 50:50 v/v. Separations were performed using the Shimadzu SPD 10A instrument using a UV-visible spectrophotometric detector. Eluent A was HPLC grade water, $\mathrm{pH} 3$, using methane sulphonic acid and eluent $\mathrm{B}$ was ammonium methane sulphonic acid (2 M), $\mathrm{pH}$ 2.5. The elution profiles were monitored by absorbance at $232 \mathrm{~nm}$. Eight commonly occurring authentic HS disaccharides (Dextra Labs) were injected and eluted with a 0-50\% linear gradient of eluent B at a flow rate of $1 \mathrm{~mL} / \mathrm{min}$ over 60 minutes. The disaccharide separation was repeated using HPLC grade water as eluent $\mathrm{A}$ and $2 \mathrm{M}$ ammonium bicarbonate as eluent $\mathrm{B}$.

Digestion of heparin. Heparin $(1 \mathrm{~g})$ was dissolved in $500 \mu \mathrm{L}$ of lyase buffer $(100 \mathrm{mM}$ sodium acetate, $10 \mathrm{mM}$ calcium acetate) and digested into oligosaccharide products using $1 \mathrm{mU}$ of heparinase I ( $1 \mathrm{mU}$ per $1 \mu \mathrm{L})$ to $10 \mathrm{mg}$ of heparin at $37^{\circ} \mathrm{C}$. At $2,3,4,6$ and 8 hours, a $120 \mu \mathrm{L}$ 
aliquot was taken from the reaction and quenched by denaturing the heparinase enzyme at $98{ }^{\circ} \mathrm{C}$ for 3 minutes. The resulting products were pooled and further separated as described below.

Size exclusion chromatography (SEC). Heparin was separated using a Biorad Econo column packed in-house with prep grade Sephadex 30 beads $(15 \mathrm{~mm}$ x $170 \mathrm{~cm}$, bead size $34 \mu \mathrm{m}$ - GE Healthcare) on a Delta 600 HPLC system (Waters). A $500 \mathrm{mg}$ sample of the pooled digested heparin, was made up to $1 \mathrm{~mL}$ with $0.5 \mathrm{M}$ ammonium bicarbonate and injected into the system. The digested heparin sample was eluted using $0.5 \mathrm{M}$ ammonium bicarbonate with a flow rate of $0.1 \mathrm{~mL} / \mathrm{min}$. The elution profile was monitored with absorbance at $232 \mathrm{~nm}$. Fractions were pooled and repeatedly freeze-dried using water until all the ammonium bicarbonate was removed.

Strong Anion-Exchange Chromatography (SAX). SEC fractions were separated using a Propac PA1 column (4.6 mm x $250 \mathrm{~mm}, 5 \mu \mathrm{m}$ bead size; Thermo Scientific) on a Delta 600 HPLC system (Waters). Eluent A was HPLC grade water and eluent B was $2 \mathrm{M} \mathrm{NaCl}$. The concentration of each oligosaccharide fraction loaded was dependent on its previous purification method, as stated in the text. The initial hexa-saccharide separation was performed on a $0-1.4 \mathrm{M}$ $\mathrm{NaCl}$ gradient over 90 minutes using the Propac PA1 column. Subsequent bespoke elution gradients for each oligosaccharide were calculated based on the first round of SAX separation. Isolated peaks were collected and subjected to further orthogonal techniques.

Oligosaccharide VSCTA-SAX chromatography. The C18 column ( $4.6 \mathrm{~mm}$ x $250 \mathrm{~mm}$, $5 \mu \mathrm{m}$ bead size - Sigma) was derivatised with $1 \mathrm{mM}$ cetyltrimethylammonium in a water: methanol ratio of 40: $60(\mathrm{v} / \mathrm{v})$. Oligosaccharides collected from the Propac PA1 SAX column separation were diluted 1 in 10 and multiple injections were performed to load the entire sample 
onto the VSCTA-SAX column. VSCTA-SAX separations were performed using a Waters Delta 600 HPLC with a UV-visible spectrophotometric detector. Eluent A was HPLC grade water and eluent $\mathrm{B}$ was $2 \mathrm{M}$ ammonium bicarbonate using a flow rate of $1 \mathrm{~mL} / \mathrm{min}$, at a temperature of 40 ${ }^{\circ} \mathrm{C}$. The elution profiles were monitored with an absorbance at $232 \mathrm{~nm}$. Gradient details for each sample are in the text and figure legends. Each fraction was dried on a SPD121B speed vac (Thermo Scientific) prior to mass spectrometry and compositional analysis.

Disaccharide compositional analysis. Purified oligosaccharide structures were digested using $10 \mu \mathrm{L}$ of $1 \mathrm{mU} / 1 \mu \mathrm{L}$, heparinase I, heparinase II and heparinase III. Each reaction was incubated at $30{ }^{\circ} \mathrm{C}$ for 24 hours to achieve complete digestion. The resulting disaccharide products were then separated on a SAX Propac PA1 (4.6 mm x $250 \mathrm{~mm}, 5 \mu \mathrm{m}$ bead (Dionex)) column using a Waters Delta 600 HPLC with a UV-visible spectrophotometric detector. Each sample was separated on a $0-1 \mathrm{M} \mathrm{NaCl}$ gradient over 60 minutes. Elution profiles were monitored with an absorbance of $232 \mathrm{~nm}$. Disaccharide standards (1 $\mu \mathrm{g}$ of each standard) were loaded on to the same SAX Propac PA1 column and separated using the same gradient, so that the samples could be compared. 
IMMS of isomeric hexasaccharides. IMMS was performed on a Synapt G1 mass spectrometer equipped with a T-wave mobility cell (Waters Corp., Milford, MA). Sample concentration was calculated based on its $232 \mathrm{~nm}$ absorbance and then adjusted to a concentration of $0.5 \mu \mathrm{M}$ in water/acetonitrile $(50 / 50 \mathrm{v} / \mathrm{v})$ with $500 \mathrm{mM}$ ammonium hydroxide.

The borosilicate tips were made in house as stated in previous publications ${ }^{38-39}$. Oligosaccharides were sprayed in a borosilicate gold coated tip and mass spectra were acquired in negative ion mode with a capillary voltage at $0.55 \mathrm{kV}$, a sample cone voltage at $7 \mathrm{~V}$, and an extraction cone voltage at $0.6 \mathrm{~V}$. The ion mobility parameters for each tetra or hexasaccharide can be found in supplementary materials. MS/MS was performed on selected ions and collisionally activated at $15 \mathrm{~V}$ and $20 \mathrm{~V}$ in the transfer cell with the mobility cell turned off in order to produce comparable CID data for each isomer.

\section{Results and discussion}

\section{Disaccharide analysis by CTA-SAX employing volatile buffers}

The natural diversity of heparin and HS provides a rich source for structure-function studies, but many oligosaccharides are isomeric structures, making purification difficult. This arises from the structures having identical negative charge, and is compounded as the chain length increases ${ }^{12,40}$. The separation problems deepen due to the molecule being linear, and the distance between the groups being close $^{41-42}$. As the number of adjacent negative charges increase, the charge-charge repulsion makes this task even more difficult. Low $\mathrm{pH}$ buffers (eg. $\mathrm{pH}$ 1) result in protonation of $50 \%$ of the sulfate groups and $99 \%$ of the carboxylic acid groups, potentially altering conformation and interaction with columns. At neutral $\mathrm{pH}$, most sulfate groups will be negatively charged causing charge-charge repulsion, as well as adduct retention. It 
is known that co-ordination of salts causes different structural conformations ${ }^{43}$, so here we used ammonia-based salts to enhance the level of diversity for isomeric separation. The other major obstacle for purification is the use of non-volatile salt buffers, which necessitates subsequent desalting methods which for hydrophilic HS oligosaccharides result in interactions and material loss (with desalting columns recoveries of only $60-70 \%$ were observed compared to $>90 \%$ recovery with the VSCTA SAX; data not shown). Non-volatile salts also affect mass spectrometry analysis, by increasing the number of adducts attached to the sulfate groups, and via signal suppression. We reasoned that the use of a volatile salt SAX method would overcome these problems. A conventional SAX method using Propac PA1 column was tested with volatile ammonium bicarbonate salts; however, neither disaccharides nor oligosaccharides eluted from the column, indicating the interaction was too strong for ammonium bicarbonate to achieve effective dissociation (data not shown). To overcome this, we investigated CTA-SAX ${ }^{19}$, in which a $\mathrm{C}-18$ reverse phase column is first derivatised using CTA, imparting a positive charge on the matrix and allowing the column to be used for SAX. Firstly, eight commonly occurring HS disaccharide standards were separated on CTA-SAX using a 0-1 M ammonium methane sulfonic acid gradient over 60 minutes (Figure 1a). All disaccharides eluted with baseline resolution; furthermore, the column separated $\alpha$ and $\beta$ anomers for the disaccharides, resulting in double peaks for many of the standards. The standards were then separated on the CTA-SAX column using the volatile ammonium bicarbonate salt (Figure 1b). As with the ammonium methane sulfonic acid, all 8 disaccharides were separated, but without separation of the anomeric structures with the minor drawback of increased baseline absorbance. The ability of the ammonium bicarbonate to dissociate disaccharides from the CTA-SAX column but not the 
Propac PA-1 column suggested that the VSCTA-SAX system might be ideal as an additional purification step for oligosaccharides.

\section{Major and minor oligosaccharide peak separation}

To prove the effectiveness of VSCTA-SAX to prevent losses, improve separation, and increase compatibility with other techniques such as mass spectrometry, we isolated a heparin hexasaccharide using traditional SEC and SAX followed by VSCTA-SAX, and determined its sequence using mass spectrometry. Full length heparin was digested with heparinase I and the oligosaccharide mixture (ranging from dp2 - dp30) was separated using SEC (data not shown). The dp6 oligosaccharide fraction isolated from SEC separation was subjected to further chromatographic separation using Propac PA1 SAX (Figure 2). Separation of the dp6 SEC fraction is shown in Figure 2; peaks A, B and C were selected for further purification. Previous work has focused on using such partially resolved SAX fractions ${ }^{44}$.

Peak A was separated further using a Propac PA1 column employing a shallower salt elution gradient to improve oligosaccharide resolution (Figure 3a). However, using this gradient full oligosaccharide separation could not be obtained. Peak D from this run was then further separated by VSCTA-SAX chromatography using ammonium bicarbonate as the elution buffer, demonstrating resolution of two separate oligosaccharide structures (Figure $3 b$ ). The reasons for these two peaks being separated could be related to the spacing of the CTA linker on the column, or because an ammonium salt is weaker in its displacement than a sodium salt. It could also be because ammonium ions are known to co-ordinate and separate oligosaccharides differently to sodium adducts as shown through graphite LC-MS (Miller, Karlsson \& Turnbull, unpublished data). The larger peak, peak E (Figure 3b), was selected for mass spectrometry analysis and 
compositional analysis to determine whether the structure was pure and establish whether it could be sequenced (Figures 3c to 3e). MS showed that this peak was pure, with a $\mathrm{m} / z$ 509.9 [M$3 \mathrm{H}]^{3-}$, which corresponds to a hexasaccharide with 6 sulfates and 1 acetyl group $\left(\mathrm{dp} 6+6 \mathrm{SO}_{3}+\right.$ 1Ac; Figure 3c). Data from tandem mass spectrometry and compositional analysis were both

used to inform sequencing (Figure $3 \mathrm{~d}$ and $3 \mathrm{e})^{45-48}$. Compositional analysis of peak E (Figure 3e), showed that this structure contains three different disaccharides: a $\Delta \mathrm{UA}$ - GlcNAc6S, a $\Delta \mathrm{UA} 2 \mathrm{~S}$ GlcNS, and a $\triangle \mathrm{UA2S}$ - GlcNS6S. The order of the disaccharides can be determined based on the acetate group, as this does not dissociate in the mass spectrometer and displays a distinct mass signature compared to sulfate groups. The ions $m / z 486\left(\mathrm{Y}_{3}, \mathrm{UA}\right.$ - GlcNAc6S - UA2S - GlcNS), $429.7\left(\mathrm{C}_{5}, \Delta \mathrm{UA2S}\right.$ - GlcNS6S - UA - GlcNAc6S - UA2S), and $636\left(\mathrm{~B}_{5}, \Delta \mathrm{UA2S}\right.$ - GlcNS6S UA - GlcNAc6S - UA2S), show that the GlcNAc is positioned in the middle of the structure and that the GlcNS is at the reducing terminal. If the GlcNAc residue was the reducing terminal disaccharide, it would be expected that ions corresponding to $m / z 536\left(\mathrm{C}_{4}\right), 615\left(\mathrm{~B}_{5}\right)$, and 624 $\left(\mathrm{C}_{5}\right)$, would be observed; none of these ions were observed in the product ion spectra. Therefore, the sequence can be defined as $\triangle \mathrm{UA2S}$ - GlcNS6S - UA - GlcNAc6S - UA2S - GlcNS (Figure S1).

\section{Separation of isomeric structures}

The majority of structures within heparin and HS have multiple isomers, due to variation in positioning of sulfate and acetate groups in different saccharides of the same mass, making isolation of single structures challenging. For example peak B (Figure 2), clearly contains multiple species and has peak shoulders, and therefore likely represents multiple oligosaccharide structures. To tackle this problem, the middle peak was collected with the aim of isolating the major product with only minor overlapping products. Peak B (Figure 2) was separated a second 
time using the SAX column with a shallower gradient $(0.6-1.1 \mathrm{M} \mathrm{NaCl}$ over 60 minutes $)$ in an effort to improve its separation (Figure 4a). In this case a greater level of separation was observed. It is likely that the resolving power of these peaks could be further enhanced by using a shallower salt gradient and reduced sample loading. Since the resulting major peak (peak F) was not baseline resolved, as commonly observed for heparin/HS oligosaccharides ${ }^{11}$, there is a need to have additional orthogonal separation methodologies. To avoid desalting and consequent losses, peak $\mathrm{F}$ (containing a high concentration of $\mathrm{NaCl}$ ) was loaded onto the VSCTA-SAX column through dilution and multiple injections. Peak F oligosaccharide structures were then eluted from the VSCTA-SAX column with a $0.7-1.4 \mathrm{M}$ ammonium bicarbonate gradient (Figure 4b). This method of separation resulted in close to base line resolution of four apparent oligosaccharide compounds, demonstrating the enhanced resolution of VSCTA-SAX chromatography. The ammonium bicarbonate salt was removed readily via centrifugal evaporation (data not shown). Peak G and peak $\mathrm{H}$ were analysed by offline ESI-MS. They both displayed a $\mathrm{m} / \mathrm{z}$ of 549.3 , confirming that they were mass isomers as anticipated, and corresponding to a dp6 with $8 \mathrm{SO}_{3}$ (Figure 4c). Furthermore, IM-MS of peak G and peak $\mathrm{H}$ revealed that they displayed different arrival time distributions (ATDs) of $3.39 \mathrm{~ms}$ and $3.67 \mathrm{~ms}$ respectively (Figure 4d). This suggests different structural conformations, with peaks G and $\mathrm{H}$ displaying more compact and extended conformations respectively. Since both compounds could be separated though a charge dependent interaction method, this result indicates that this separation is at least partly dependent on their different conformational properties.

To further determine the structure of the isolated peaks, tandem mass spectrometry and compositional analysis were performed on both peak $\mathrm{G}$ and peak $\mathrm{H}$. MS revealed very different product ion spectra (Figure 4e). Compositional analysis showed that peak $\mathrm{G}$ contained a single 
$\Delta \mathrm{UA}$ - GlcNS6S and two $\triangle \mathrm{UA2S}$ - GlcNS6S disaccharides, whereas peak H contained one $\Delta \mathrm{UA2S}$ - GlcNS and two $\triangle \mathrm{UA2S}$ - GlcNS6S (Figure 4f). Since there are two $\triangle \mathrm{UA} 2 \mathrm{~S}$-GlcNS6S, the first piece of information to identify in the product ion spectra is whether these disaccharides are adjacent. In peak G no doubly charged 576 product ion was observed (Figure 4e), whereas in peak $\mathrm{H}$ a doubly charged 576 product ion was observed, indicating that the two tri-sulfated disaccharides must be adjacent. Therefore, the sequence of peak H is $\triangle \mathrm{UA2S}$ - GlcNS - UA2S GlcNS6S - UA2S - GlcNS6S or $\triangle \mathrm{UA2S}$ - GlcNS6S - UA2S - GlcNS6S - UA2S - GlcNS (Figure S-1 and S-2). In contrast, Peak G has a major triply charged product ion of 464 corresponding to $\mathrm{Z}_{4}$ (GlcNS6S - UA - GlcNS6S - UA2S - GlcNS6S). The $\mathrm{Y}_{3}$ ion (UA - GlcNS6S - UA2S GlcNS6S) and $Y_{2}$ ion (GlcNS6S - UA2S - GlcNS6S) confirmed that the UA - GlcNS6S is in the middle of the oligosaccharide structure (Figure 4e). Therefore, the sequence of the oligosaccharide isolated from peak G is $\triangle \mathrm{UA2S}$ - GlcNS6S - UA - GlcNS6S - UA2S - GlcNS6S (Figures S-1 and S-2).

In a further demonstration of the utility of VSCTA-SAX chromatography, we were also able to separate another three hexasaccharides $\left(\mathrm{dp} 6+8 \mathrm{SO}_{3}\right)$ from the same dp6 SEC fraction (peak C; Figure 2). This peak was further separated on a shallow gradient $(0.84-1.2 \mathrm{M} \mathrm{NaCl}$ over 60 minutes) on a SAX Propac PA1 column (Figure 5a). The structures were separated into two peaks, I and J (although not to baseline resolution) and were collected separately. It should be noted that collecting partial peaks and re-separating on a shallower gradient can also lead to improved resolving power. Peak I and peak J were both subjected to separation on the VSCTASAX column with a $0.8 \mathrm{M}-1.5 \mathrm{M}$ ammonium bicarbonate gradient. This resulted in improvements in peak resolution (Figure 5b and 5c). MS showed that all three structures had the same $m / z$ ratio of $549.3[\mathrm{M}-3 \mathrm{H}]^{3-}$ (data not shown). It was particularly noteworthy that IMMS on 
these three isomeric structures revealed that each structure had different ATDs. Peak K displayed the most extended conformation with an ATD of $3.74 \mathrm{~ms}$, peak M a slightly more compact conformation $(3.60 \mathrm{~ms})$, and peak $\mathrm{L}$ a much more compact structural conformation $(3.46 \mathrm{~ms})$ (Figure 5d). MS/MS and compositional analysis was completed on all three of these structures (Figure 5e and 5f). Compositional analysis showed that peak $\mathrm{K}$ and peak $\mathrm{L}$ each contained one $\Delta \mathrm{UA}$ - GlcNS6S and two $\triangle \mathrm{UA2S}$ - GlcNS6S disaccharides whereas peak M contained one $\Delta \mathrm{UA2S}$ - GlcNS and two $\triangle \mathrm{UA2S}$ - GlcNS6S disaccharides. MSMS of these structures showed that all three contained a doubly charged product ion of 576 corresponding to an intact dp4 + $6 \mathrm{SO}_{3}$ (UA2S - GlcNS6S - UA2S - GlcNS6S). Thus the sequence of peaks $\mathrm{K}$ and $\mathrm{L}$ are defined as $\Delta \mathrm{UA}$ - GlcNS6S - UA2S - GlcNS6S - UA2S - GlcNS6S and $\Delta \mathrm{UA2S}$ - GlcNS6S - UA2S GlcNS6S - UA - GlcNS6S respectively and the sequence of peak M / peak H is $\triangle \mathrm{UA2S}$ GlcNS6S - UA2S - GlcNS6S - UA2S - GlcNS or $\triangle \mathrm{UA2S}$ - GlcNS - UA2S - GlcNS6S - UA2S GlcNS6S (Figure S-1). These two structures with identical compositional analysis but, disaccharide UA - GlcNS6S at either the reducing end or the non-reducing end, displayed the largest ATD difference (3.74 ms and $3.46 \mathrm{~ms})$. This suggests that oligosaccharide conformation can have an effect on separations using SAX columns, possibly via the different distribution of free electrons affecting the strength of the interaction.

\section{Conclusions}

Here we demonstrate the isolation, separation and analysis of multiple hexasaccharide structures from heparin oligosaccharide starting material. The VSCTA-SAX methodology we describe resulted in enhanced resolution compared to previous methods, likely resulting from a combination of the weaker displacement salt, the co-ordination of ammonium with the sulfate groups and differences in structural conformation and electron distribution. We have 
demonstrated that VSCTA-SAX provides improved separation of structural isomers compared to HILIC and RP-IP, and also removes the need for traditional desalting methods. Volatile salt methods for heparin/HS chromatography techniques are increasingly in demand to improve yields, which is crucial since only very small amounts of individual structures are often purified from complex starting mixtures. Thus the practicality of subsequent analysis and screening is enhanced using VSCTA-SAX. We demonstrate here that the combination of established SEC and SAX separation techniques with VSCTA-SAX allowed the separation of 5 isomeric structures $\left(\mathrm{dp} 6+8 \mathrm{SO}_{3}\right)$ and their sequence determination in a manner not possible with traditional methods alone. We have also applied the method to purification of isomeric heparin saccharides for studying selectivity of interactions with the chemokine MCP-1/CCL2 (Miller et al, submitted to Analytical Chemistry). We conclude that VSCTA-SAX is a powerful additional tool to enhance structure-activity studies on heparin/HS saccharides. 


\section{References}

1. Puvirajesinghe, T. M.; Turnbull, J. E. Metabolites 2012, 2, 1060-89.

2. $\quad$ Farrugia, B. L.; Lord, M. S.; Melrose, J.; Whitelock, J. M. Molecules 2015, 20, 4254-76.

3. Arungundram, S.; Al-Mafraji, K.; Asong, J.; Leach, F. E., 3rd; Amster, I. J.; Venot, A.;

Turnbull, J. E.; Boons, G. J. J Am Chem Soc 2009, 131, 17394-405.

4. Dulaney, S. B.; Xu, Y.; Wang, P.; Tiruchinapally, G.; Wang, Z.; Kathawa, J.; El-

Dakdouki, M. H.; Yang, B.; Liu, J.; Huang, X. J Org Chem 2015, 80, 12265-79.

5. Schworer, R.; Zubkova, O. V.; Turnbull, J. E.; Tyler, P. C. Chemistry 2013, 19, 6817-23.

6. Saad, O. M.; Ebel, H.; Uchimura, K.; Rosen, S. D.; Bertozzi, C. R.; Leary, J. A.

Glycobiology 2005, 15, 818-26.

7. Wei, W.; Miller, R. L.; Leary, J. A. Anal Chem 2013, 85, 5917-23.

8. Wei, W.; Ninonuevo, M. R.; Sharma, A.; Danan-Leon, L. M.; Leary, J. A. Anal Chem 2011, $83,3703-8$.

9. Allen, B. L.; Rapraeger, A. C. J Cell Biol 2003, 163, 637-48.

10. Ori, A.; Free, P.; Courty, J.; Wilkinson, M. C.; Fernig, D. G. Mol Cell Proteomics 2009, $8,2256-65$.

11. Powell, A. K.; Ahmed, Y. A.; Yates, E. A.; Turnbull, J. E. Nat Protoc 2010, 5, 821-33.

12. Hileman, R. E.; Smith, A. E.; Toida, T.; Linhardt, R. J. Glycobiology 1997, 7, 231-9.

13. Ziegler, A.; Zaia, J. J Chromatogr B Analyt Technol Biomed Life Sci 2006, 837, 76-86.

14. Staples, G. O.; Bowman, M. J.; Costello, C. E.; Hitchcock, A. M.; Lau, J. M.; Leymarie, N.; Miller, C.; Naimy, H.; Shi, X.; Zaia, J. Proteomics 2009, 9, 686-95. 
15. Linhardt, R. J.; Gu, K. N.; Loganathan, D.; Carter, S. R. Anal Biochem 1989, 181, 288-

96.

16. Thanawiroon, C.; Linhardt, R. J. J Chromatogr A 2003, 1014, 215-23.

17. Grimshaw, J. Electrophoresis 1997, 18, 2408-14.

18. Mao, W.; Thanawiroon, C.; Linhardt, R. J. Biomed Chromatogr 2002, 16, 77-94.

19. Vives, R. R.; Goodger, S.; Pye, D. A. Biochem J 2001, 354, 141-7.

20. Mourier, P. A.; Viskov, C. Anal Biochem 2004, 332, 299-313.

21. Mourier, P. A.; Agut, C.; Souaifi-Amara, H.; Herman, F.; Viskov, C. J Pharm Biomed Anal 2015, 115, 431-42.

22. El Rassi, Z.; Mechref, Y. Electrophoresis 1996, 17, 275-301.

23. Lee, G. J.; Evans, J. E.; Tieckelmann, H.; Dulaney, J. T.; Naylor, E. W. Clin Chim Acta 1980, $104,65-75$.

24. Kuberan, B.; Lech, M.; Zhang, L.; Wu, Z. L.; Beeler, D. L.; Rosenberg, R. D. J Am Chem Soc 2002, 124, 8707-18.

25. Toyoda, H.; Kinoshita-Toyoda, A.; Fox, B.; Selleck, S. B. J Biol Chem 2000, 275, 21856-61.

26. Vongchan, P.; Warda, M.; Toyoda, H.; Toida, T.; Marks, R. M.; Linhardt, R. J. Biochim Biophys Acta 2005, 1721, 1-8.

27. Mousa, S. A.; Zhang, F.; Aljada, A.; Chaturvedi, S.; Takieddin, M.; Zhang, H.; Chi, L.;

Castelli, M. C.; Friedman, K.; Goldberg, M. M.; Linhardt, R. J. J Clin Pharmacol 2007, 47, 1508-20.

28. Hitchcock, A. M.; Bowman, M. J.; Staples, G. O.; Zaia, J. Electrophoresis 2008, 29, 4538-48. 
29. Royle, L.; Roos, A.; Harvey, D. J.; Wormald, M. R.; van Gijlswijk-Janssen, D.; Redwan el, R. M.; Wilson, I. A.; Daha, M. R.; Dwek, R. A.; Rudd, P. M. J Biol Chem 2003, 278, 2014053.

30. Akiyama, H.; Shidawara, S.; Mada, A.; Toyoda, H.; Toida, T.; Imanari, T. J Chromatogr 1992, 579, 203-7.

31. Saitoh, H.; Takagaki, K.; Majima, M.; Nakamura, T.; Matsuki, A.; Kasai, M.; Narita, H.; Endo, M. J Biol Chem 1995, 270, 3741-7.

32. Hitchcock, A. M.; Yates, K. E.; Costello, C. E.; Zaia, J. Proteomics 2008, 8, 1384-97.

33. Shi, X.; Zaia, J. J Biol Chem 2009, 284, 11806-14.

34. Naimy, H.; Leymarie, N.; Bowman, M. J.; Zaia, J. Biochemistry 2008, 47, 3155-61.

35. Naimy, H.; Leymarie, N.; Zaia, J. Biochemistry 2010, 49, 3743-52.

36. Dreyfuss, J. M.; Jacobs, C.; Gindin, Y.; Benson, G.; Staples, G. O.; Zaia, J. Anal Bioanal Chem 2011, 399, 727-35.

37. Galeotti, F.; Volpi, N. J Chromatogr A 2016, 1445, 68-79.

38. Leary, J. A.; Miller, R. L.; Wei, W.; Schworer, R.; Zubkova, O. V.; Tyler, P. C.;

Turnbull, J. E. Eur J Mass Spectrom 2015, 21, 245-54.

39. Dykstra, A. B.; Sweeney, M. D.; Leary, J. A. Biomolecules 2013, 3, 905-22.

40. Pervin, A.; Gallo, C.; Jandik, K. A.; Han, X. J.; Linhardt, R. J. Glycobiology 1995, 5, 8395.

41. Mulloy, B.; Johnson, E. A. Carbohydr Res 1987, 170, 151-65.

42. Mulloy, B.; Forster, M. J. Glycobiology 2000, 10, 1147-56.

43. Seo, Y.; Schenauer, M. R.; Leary, J. A. Int J Mass Spectrom 2011, 303, 191-198.

44. Guimond, S. E.; Turnbull, J. E. Curr Biol 1999, 9, 1343-6. 
45. Saad, O. M.; Leary, J. A. J Am Soc Mass Spectrom 2004, 15, 1274-86.

46. Saad, O. M.; Leary, J. A. Anal Chem 2005, 77, 5902-11.

47. Huang, Y.; Yu, X.; Mao, Y.; Costello, C. E.; Zaia, J.; Lin, C. Anal Chem 2013, 85, 11979-86.

48. Kailemia, M. J.; Li, L.; Xu, Y.; Liu, J.; Linhardt, R. J.; Amster, I. J. Mol Cell Proteomics 2013, 12, 979-90.

\section{Acknowledgements}

Financial support for this research was provided by a $\mathrm{PhD}$ studentship to RM from the Medical Research Council (MRC) and Engineering and Physical Sciences Research (EPSRC) Council UK (UoL/MRC-EPSRC), a Senior Research Fellowship from the MRC to JET (G117/423), a Biotechnology and Biological Sciences Research Council project grant to JET (BB/I004343/1) and NIH project grant to JAL (GM47356-20).

Supporting Information Available: Figure S-1. Structural representation of each sequenced heparin saccharide; Figure S-2. A workflow procedure involved with the purification and sequencing of oligosaccharides; Supporting methods (4 pages). This material is available free of charge via the Internet at http://pubs.acs.org.

Figure 1. CTA-SAX separation of HS standards using non-volatile and volatile buffers. Disaccharide standards were injected and eluted from CTA-derived Discovery C18 column using a 0-1 M gradient over 60 minutes of: a) ammonium methane sulfonic acid, $\mathrm{pH}$ 2.5. b) ammonium bicarbonate. Standards: $1-\Delta$ UA-GlcNAc, $2-\Delta$ UA-GlcNS, $3-\Delta$ UA-GlcNAc6S, $4-\Delta U A 2 S-$ GlcNAc, $5-\Delta$ UA-GlcNS6S, $6-\Delta$ UA2S-GlcNS, $7-\Delta$ UA2S-GlcNAc6S, $8-\Delta$ UA2SGlcNS6S. 
Figure 2. SAX separation of a heparin hexasaccharide mixture. The dp6 oligosaccharide fraction isolated from SEC (data not shown) was collected and separated using a Propac PA1 SAX column on a 0-1.4 M NaCl gradient over 90 minutes as described in Methods. Arrowed peaks were collected for further separation using subsequent methodologies.

Figure 3. Purification of minor and major oligosaccharide products using SAX and VSCTA-SAX. a) SAX separation of peak A on a Propac PA1 SAX column using a $0.6-1.1 \mathrm{M}$ $\mathrm{NaCl}$ gradient over 60 minutes. b) Peak D was separated further using a VSCTA-SAX column on a $0.7-1.4 \mathrm{M}$ ammonium bicarbonate gradient B in A over 60 minutes. c) MS of peak E, showing a $m / z$ of 509.9 which corresponds to dp6 $+6 \mathrm{SO}_{3}+1 \mathrm{Ac}$. d) MS/MS of peak E at $15 \mathrm{~V}$ and $20 \mathrm{~V}$. e) Disaccharide analysis of peak E. The lower chromatograms correlates to disaccharide standards. The upper trace is disaccharide analysis of peak E. This information defined the structure an overall sequence of $\triangle \mathrm{UA2S}$ - GlcNS6S - UA - GlcNAc6S - UA2S GlcNS. Standards: 3 - $\Delta$ UA - GlcNAc6S, 4 - $\Delta$ UA2S - GlcNAc, $5-\Delta U A$ - GlcNS6S, $6-$ $\Delta \mathrm{UA} 2 \mathrm{~S}$ - GlcNS, 7 - $\Delta \mathrm{UA2S}$ - GlcNAc6S, 8 - $\Delta \mathrm{UA2S}$ - GlcNS6S.

Figure 4. Purification and analysis of isomeric heparin hexasaccharide products from Peak B (Figure 2) using SAX and VSCTA-SAX. a) SAX separation of peak B on a Propac PA1 SAX column using a $0.6-1.1 \mathrm{M} \mathrm{NaCl}$ gradient over 60 minutes. b) Peak F was separated further using a VSCTA-SAX column on a $0.7-1.4 \mathrm{M}$ ammonium bicarbonate gradient over 60 minutes. c) MS of peak $\mathrm{G}$ and $\mathrm{H}$, displayed a $m / z$ of 549.3 , which corresponds to dp6 $+8 \mathrm{SO}_{3}$. d) IMMS of peak $\mathrm{G}$ and peak H. e) MS/MS of peak $\mathrm{G}$ and peak $\mathrm{H}$ at $15 \mathrm{~V}$. f) The lower chromatogram correlates to disaccharide standards, the upper two chromatograms correlates to disaccharide analysis of peak $\mathrm{G}$ and peak $\mathrm{H}$. This information gave peak $\mathrm{G}$ an overall sequence of $\Delta \mathrm{UA} 2 \mathrm{~S}$ GlcNS6S - UA - GlcNS6S - UA2S - GlcNS6S and peak H a sequence of $\Delta$ UA2S - GlcNS - 
UA2S - GlcNS6S - UA2S -GlcNS6S. Standards: 3 - $\Delta$ UA - GlcNAc6S, 4 - $\Delta$ UA2S - GlcNAc, 5 - $\Delta \mathrm{UA}$ - GlcNS6S, $6-\Delta \mathrm{UA2S}$ - GlcNS, 7 - $\Delta \mathrm{UA2S}$ - GlcNAc6S, 8 - $\Delta \mathrm{UA2S}$ - GlcNS6S.

Figure 5. Separation of three isomeric structures. a) Propac PA1 SAX separation of peak C on a $0.84 \mathrm{M}-1.2 \mathrm{M} \mathrm{NaCl}$ gradient over 60 minutes. b) VSCTA-SAX separation of peak I on a $0.8 \mathrm{M}-1.5 \mathrm{M}$ ammonium bicarbonate gradient over 60 minutes, isolating peak K. c) VSCTASAX separation of peak $\mathrm{J}$ on a $0.8 \mathrm{M}-1.5 \mathrm{M}$ ammonium bicarbonate gradient over $60 \mathrm{~min}$, isolating peak L and peak M. d) IMMS separation of peaks K, L and M. e) MS/MS of peaks K, L and $\mathrm{M}$ at $15 \mathrm{~V}$. f) The lower chromatogram correlates to disaccharide standards, the upper three chromatograms correlates to disaccharide analysis of peaks $\mathrm{K}, \mathrm{L}$ and $\mathrm{M}$. The sequence of peak $\mathrm{K}$ is $\triangle \mathrm{UA}$ - GlcNS6S - UA2S - GlcNS6S - UA2S - GlcNS6S, peak L is $\triangle \mathrm{UA2S}$ - GlcNS6S - UA2S - GlcNS6S - UA - GlcNS6S, and peak M is $\triangle \mathrm{UA2S}$ - GlcNS6S - UA2S - GlcNS6S - UA2S GlcNS. Standards: $3-\Delta \mathrm{UA}$ - GlcNAc6S, $4-\Delta \mathrm{UA2S}$ - GlcNAc, 5 - $\Delta \mathrm{UA}-$ GlcNS6S, $6-$ $\Delta \mathrm{UA2S}$ - GlcNS, 7 - $\Delta \mathrm{UA2S}$ - GlcNAc6S, 8 - $\Delta \mathrm{UA2S}$ - GlcNS6S. 

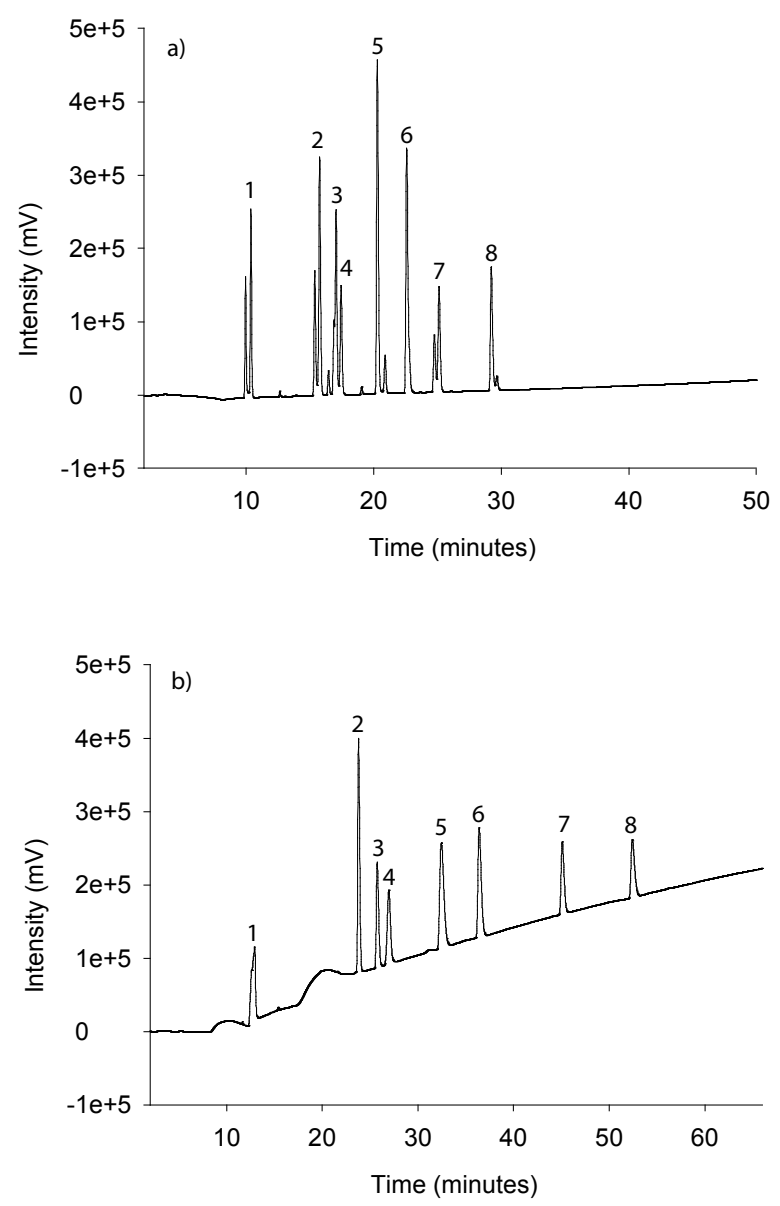

Figure 1. CTA-SAX separation of HS standards using non-volatile and volatile buffers. 


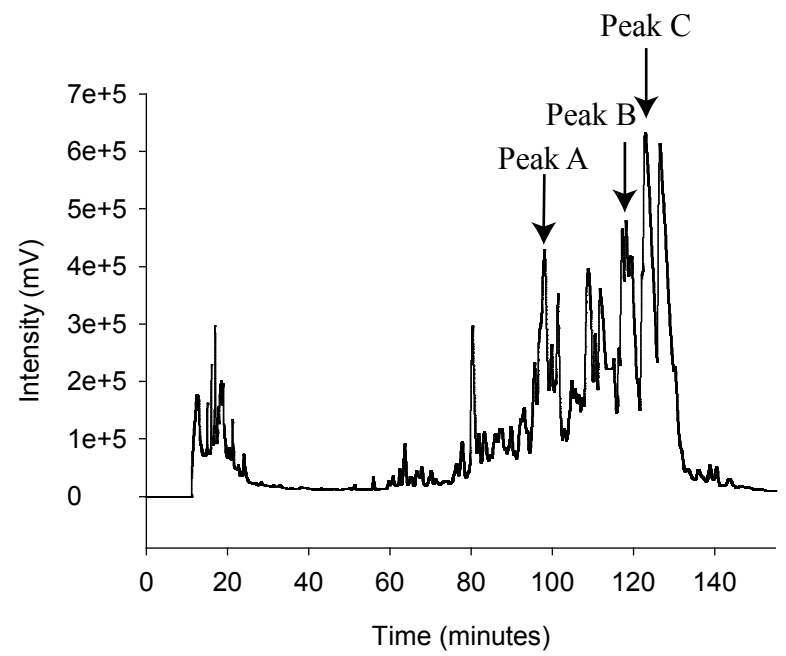

Figure 2. SAX separation of a heparin hexasaccharide mixture. 

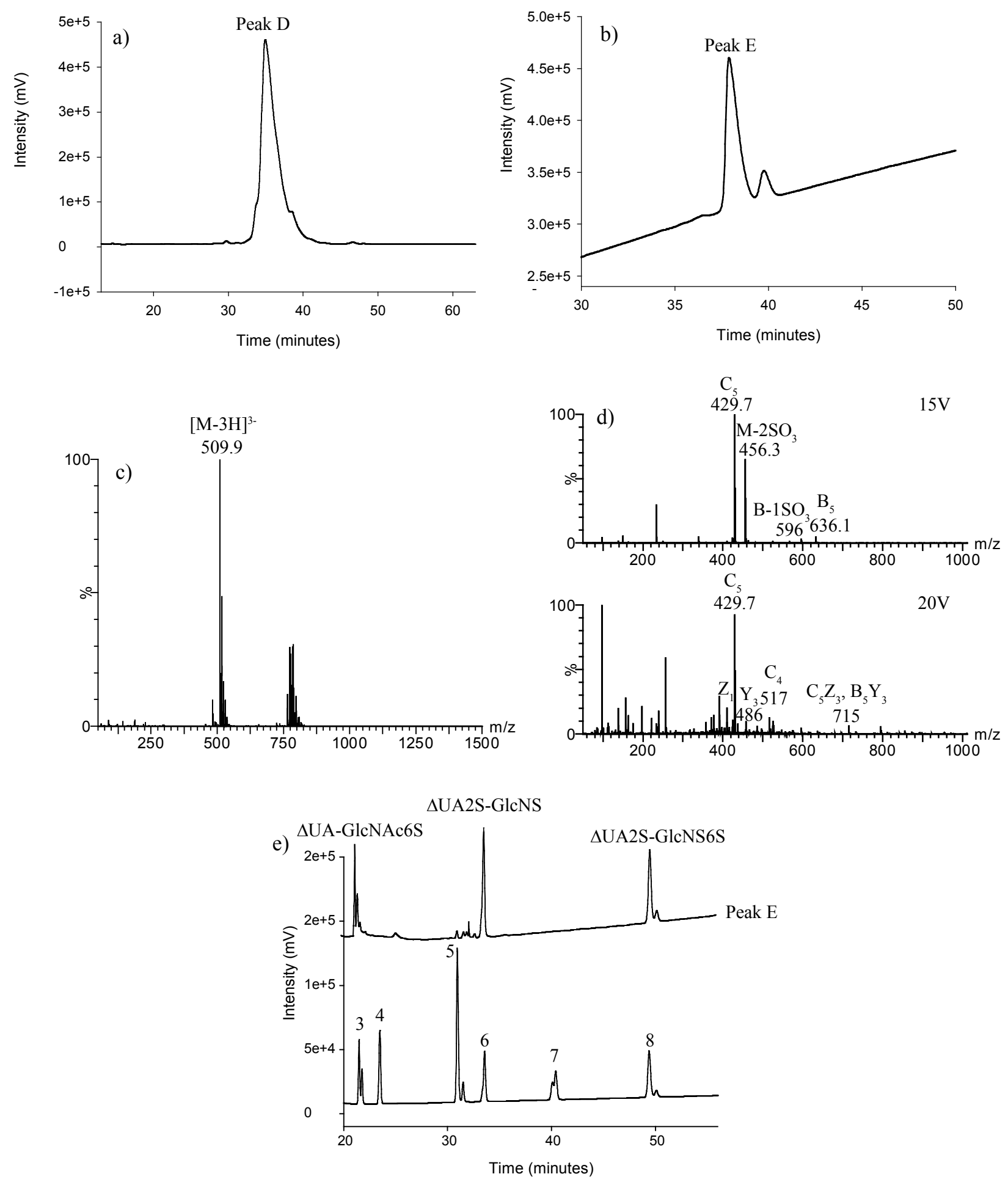

Figure 3. Purification of minor and major oligosaccharide products using SAX and VSCTA-SAX. 

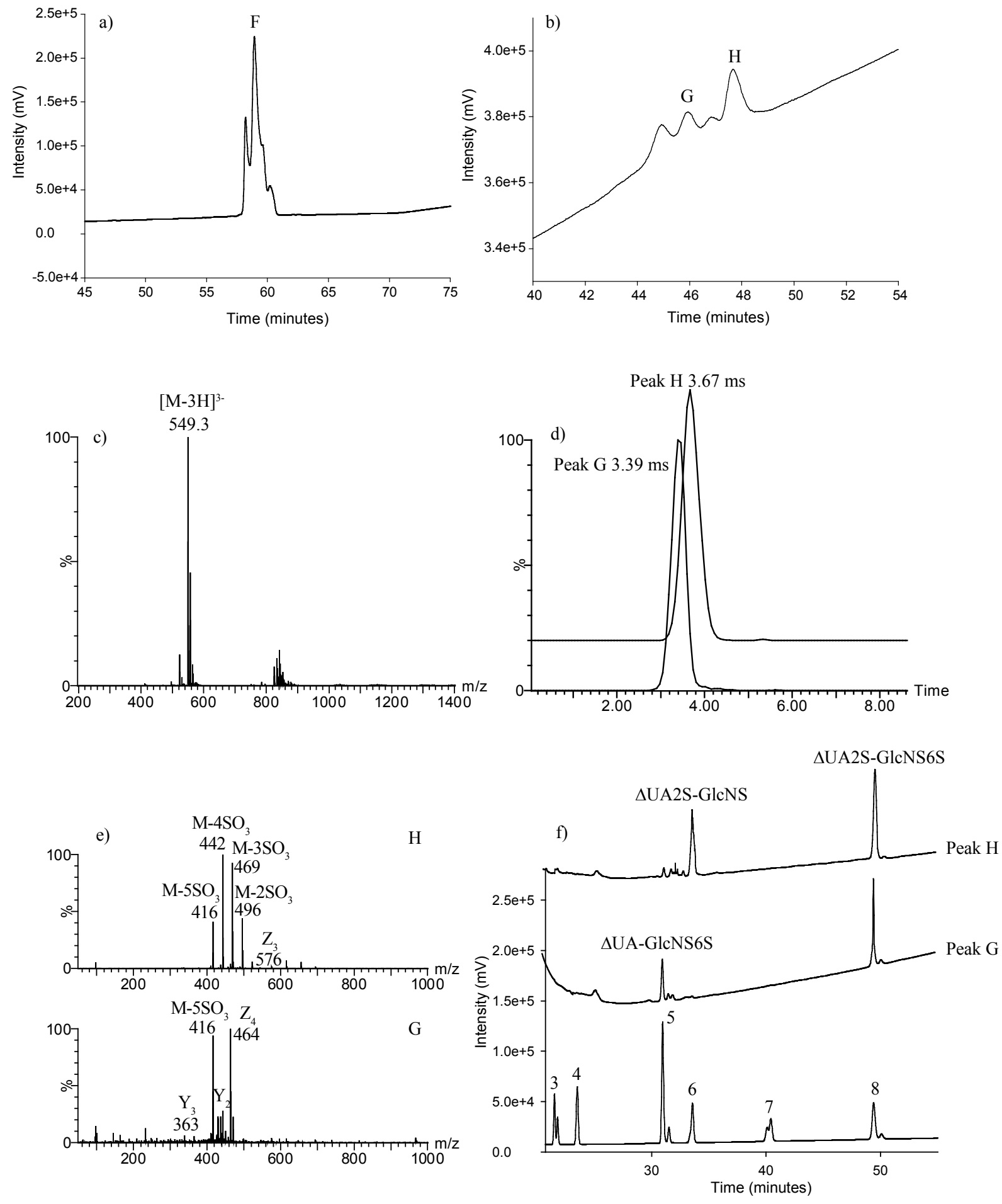

Figure 4. Purification and analysis of isomeric heparin hexasaccharide products from Peak B (Figure 2) using SAX and VSCTA-SAX. 

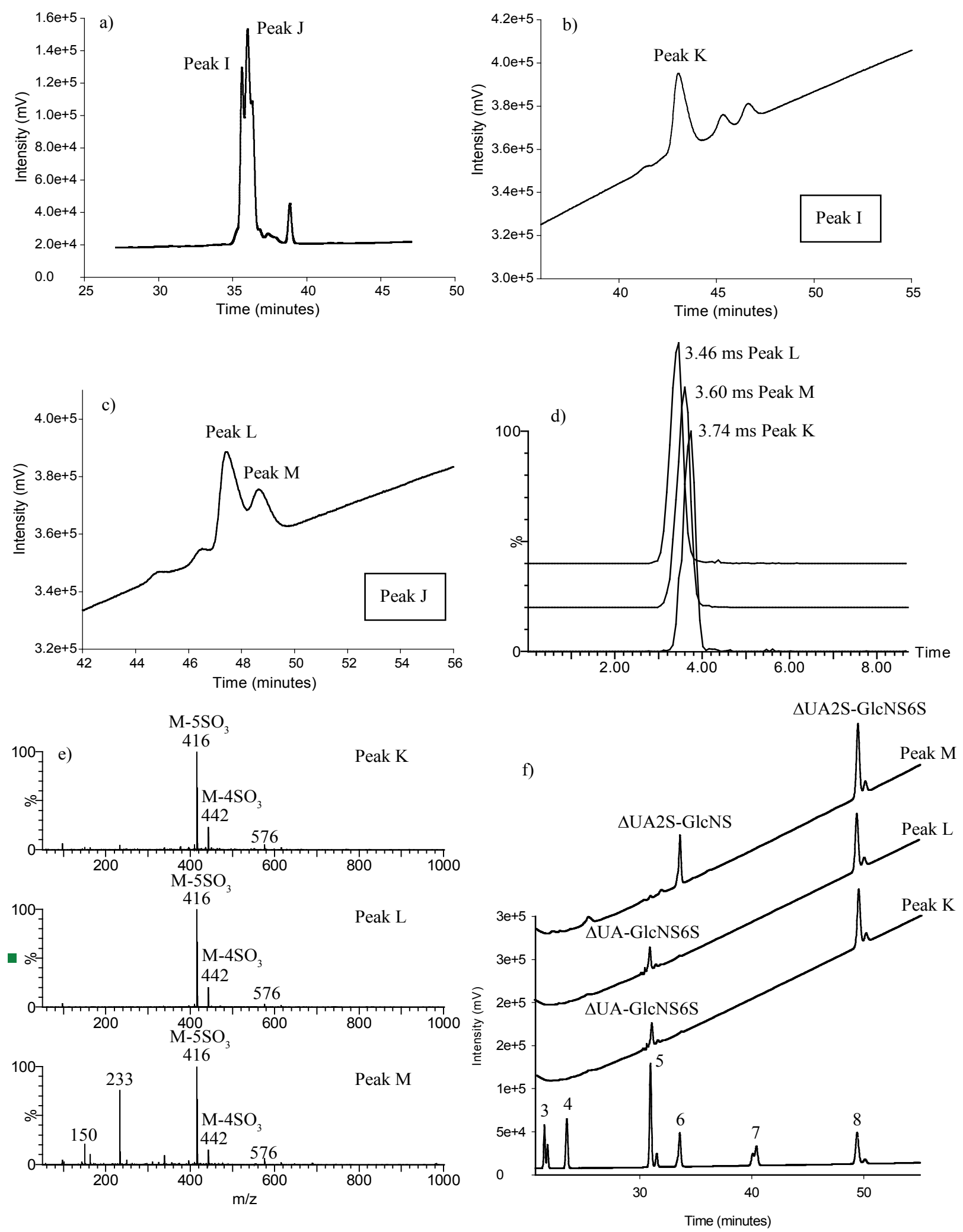

Figure 5. Separation of three isomeric structures. 